\title{
Ciências da Educação e Ciências Cognitivas. Contributos para uma abordagem transdisciplinar
}

\author{
Teresa N. R. Gonçalves \\ Universidade Nova de Lisboa, Portugal
}

\begin{abstract}
Resumo
O desenvolvimento da investigação científica no domínio das Ciências Cognitivas tem contribuído para uma nova e mais complexa perspectiva sobre o sujeito da educação, a cognição e a aprendizagem. A natureza do fenómeno educativo e as rápidas transformações a que tem estado sujeito remetem para a necessidade de incorporar uma teoria da mudança que permita lidar com as múltiplas questões e problemas com que se confronta a reflexão em educação e a prática educativa. Estes aspectos tornam necessários novos paradigmas de investigação e novas formas de colaboração que ultrapassem as barreiras entre os saberes. Partindo da teoria da complexidade como paradigma emergente, quer no âmbito das Ciências Naturais quer das Ciências Sociais e Humanas, apresentam-se alguns argumentos a favor da colaboração entre Ciências da Educação e Ciências Cognitivas, clarificando a sua necessidade e implicações, assim como as possibilidades que representa para a investigação e acção educativas.
\end{abstract}

Palavras-chave

Educação; Cognição; Complexidade; Transdisciplinaridade

\section{O campo epistemológico das Ciências da Educação e a investigação em Ciências Cognitivas}

Em primeiro lugar, importa esclarecer que o campo das Ciências da Educação é, desde a sua origem, pela sua própria natureza, complexidade, 
abrangência e importância dos fenómenos que trata e das questões com que se confronta, um campo de cruzamento natural de diferentes disciplinas e metodologias. O estudo sobre a educação dos seres humanos é um campo atravessado, desde sempre, por perplexidades e tensões, assim como por uma multiplicidade de pontos de vista que o tornam uma área em permanente construção e reconstrução. Dentro deste campo, a dimensão prática da acção humana cruza-se e confronta-se com a reflexão sobre os meios e fins da educação, a partir da e sobre a própria acção educativa.

Entende-se que a complexidade dos fenómenos humanos a que chamamos educação tem múltiplas dimensões e pode, por isso, ser tratada a partir múltiplos pontos de vista, para além das diferentes organizações epistemológicas e estratificação dos saberes vigentes (Morin, 1999). O que dá unidade, coerência e razão de ser à existência de um campo de conhecimento designado como Ciências da Educação e de uma área de conhecimento chamada teoria da educação é a sua estruturação em torno de grandes eixos e questões fundamentais (García Carrasco, 2007; Vazquéz, 2003), a partir das quais se deve estruturar a investigação nesse campo de conhecimento. Neste sentido, o campo epistemológico da educação seria, como refere Roldão (2005, p. 15):

(...) uma ciência ou campo científico em construção - Educação - definido epistemologicamente pela natureza educacional das suas questões, mobilizador de outros campos para a clarificação dessas questões, e praticando metodologias e conceptualizações não apenas importadas das Ciências Sociais, mas cada vez mais específicas face à natureza do campo epistemológico, que assim se irá consolidando e unificando transversalmente as suas múltiplas vertentes (...).

Enquanto realidade em construção, o campo educativo deve abrir-se à reflexão sobre questões e conhecimentos emergentes noutros campos de investigação. Poderá, desta forma, complementar e (re)orientar os temas, problemas e perspectivas que enquadram o seu âmbito científico, tendo em conta que o fenómeno educativo é multidimensional e complexo, e que o campo epistemológico das Ciências da Educação está sujeito à problematização tanto interna como externa.

As Ciências Cognitivas constituem um campo pluridisciplinar, onde convergem distintas aproximações ao estudo da cognição. O estudo sobre a cognição pode ser agrupado em diferentes categorias que correspondem a 
domínios de investigação dentro das Ciências Cognitivas e que incluem: a percepção, a memória, a aprendizagem, a linguagem, a inteligência, o raciocínio, a consciência, os processos de atenção, a criatividade, o desenvolvimento, a acção e seus mecanismos, as emoções. Actualmente, as Ciências Cognitivas englobam um conjunto de pesquisas que se relacionam com diferentes disciplinas: a Psicologia Cognitiva, a Inteligência Artificial, as Neurociências, a Linguística, a Filosofia da Mente e, mais recentemente, a Antropologia Cognitiva e a Sociologia Cognitiva, a Biologia Evolutiva (Dortier, 2003), fundadas numa abordagem pluridisciplinar da cognição e seus mecanismos. Entende-se por Ciências Cognitivas, não só as abordagens relacionadas com o chamado cognitivismo clássico ${ }^{1}$, que entendem a mente como um processador de informação, mas também outras abordagens científicas que assumem uma perspectiva mais integradora da cognição, como as Neurociências Cognitivas (Damásio, 2001; Gazzaniga, 2004) ou a Biologia Evolutiva (Lewontin, 1998).

A relação intrínseca entre cognição e aprendizagem - a cognição subjaz à aprendizagem, tal como a aprendizagem é susceptível de modificar as estruturas cognitivas - torna, desde logo, patente a proximidade dos dois campos epistemológicos e as intersecções entre eles. Existe, desde sensivelmente meados do século passado, um reconhecimento progressivo da importância da investigação em Ciências Cognitivas no campo da educação, tanto pela incorporação desses conhecimentos e teorias no discurso e práticas educativas, como pelo surgimento de propostas de promoção cognitiva (programas de 'a pensar' e de 'aprender a aprender') implementadas no campo educativo e que têm como base os conhecimentos e teorias originários do campo das Ciências Cognitivas. No entanto, essa influência tem-se reflectido apenas de forma parcial, e por vezes contraditória (Davis, Sumara, \& Luce-Kapler, 2008), no campo da educação. Tem estado relacionada essencialmente com os contributos da Psicologia do Desenvolvimento e da Psicologia Cognitiva (Cruz \& Fonseca, 2002) e tem-se centrado fundamentalmente em abordagens relacionadas com a teoria do processamento da informação (processos cognitivos e metacognitivos) e em aproximações desenvolvimentalistas ao estudo da cognição.

A recente evolução das investigações em Ciências Cognitivas, através da incorporação de novos campos científicos, assim como os novos 
conhecimentos sobre o cérebro e sobre o desenvolvimento emergentes das Neurociências Cognitivas e da Biologia Evolutiva, tem contribuído para um novo entendimento da cognição e seus mecanismos. Considera-se que estes conhecimentos têm importantes implicações para a compreensão dos processos de aprendizagem e dos factores que influenciam o desenvolvimento cognitivo dos indivíduos, na medida em que contribuem para a compreensão da complexidade do pensamento. Essas implicações devem ser integradas no âmbito do discurso sobre a educação e nas práticas educativas. Eles podem contribuir para uma perspectiva mais integradora da cognição e da aprendizagem e, como tal, devem ser enquadrados no âmbito da reflexão sobre a educação.

\section{Educação, cognição e complexidade}

O paradigma da complexidade tem emergido, nas últimas décadas, como referente da reflexão e investigação quer no âmbito das Ciências Naturais quer no das Ciências Sociais e Humanas². Em termos epistemológicos, o debate sobre a complexidade tem evidenciado a necessidade de novas ferramentas de compreensão do mundo, do fenómeno educativo e do sujeito a educar, que possibilitem uma aproximação sistémica ao estudo da educação ${ }^{3}$. A inadequação das práticas educativas relativamente à dinâmica da cognição humana (Dumont, Istance, \& Benavides, 2010), dos currículos relativamente à actualidade, à representação e à organização das disciplinas (Osberg, Biesta, \& Cilliers, 2008), o fracasso das estruturas escolares no que se refere à sua adaptação à crescente diversidade das populações que servem e à dinâmica dos seus contextos (Bruer, 1997), assim como as questões relacionadas com a formação, prática e desenvolvimento profissional dos professores (Roldão, 2007; Flores \& Day, 2006), demonstram, como referem Jörg, Davis, e Nickmans (2007), que os paradigmas vigentes em educação se baseiam num pensamento linear e têm negligenciado a complexidade inerente à realidade educativa. Nesta medida, têm sido incapazes de oferecer um entendimento em profundidade desta realidade. São, por isso, necessárias abordagens que consigam dar conta da complexidade inerente aos processos educativos, centrando-se na sua natureza transfenomenal (Davis, 2008) e assentes no exercício da transdisciplinaridade ${ }^{4}$ e da trans ou interdiscursividade ${ }^{5}$, que se 
traduzam em novas formas de investigação, novos conceitos e novas práticas de avaliação. O pensamento que toma como referência a complexidade centra-se nesta transfenomenalidade entendida como a forma como determinados fenómenos emanam de outros fenómenos e, por sua vez, se desdobram eles próprios noutros fenómenos.

No âmbito da educação, a relevância da teoria da complexidade resulta da possibilidade que representa para a superação de algumas metáforas mecanicistas dominantes e da sua substituição por teorias mais orgânicas que permitam, entre outros aspectos, uma nova compreensão e explicação dos processos e mecanismos de aprendizagem e de funcionamento e desenvolvimento cognitivo (Gonçalves, 2008). A complexidade permitiria superar a ideia tradicional de causalidade e dispensar as abordagens mecanicistas na medida em que representa um movimento transdisciplinar que assenta no reconhecimento da inutilidade destas abordagens para a compreensão e previsão de fenómenos como a evolução dos ecossistemas, o funcionamento e desenvolvimento do cérebro ou os fenómenos sociais em larga escala.

A formalização da ciência da complexidade como campo do saber resulta da constatação de que os investigadores nas várias disciplinas foram desenvolvendo métodos e explicações que se focavam, não em relações lineares, mas em processos simultaneamente imprevisíveis e padronizados (Jörg et al., 2007). No centro das suas preocupações está a natureza complexa, multidimensional, auto-organizativa e auto-transformativa de determinados fenómenos, entre eles o fenómeno educativo. Os fenómenos complexos são espontâneos, imprevisíveis, irredutíveis, contextuais. São sistemas adaptativos - daí a afirmação de que são sistemas que aprendem -, e evolutivos, o que os torna irredutíveis a explicações mecanicistas (Lewontin, 1998). No quadro desta perspectiva, saber, aprender e ensinar são fenómenos complexos.

No âmbito educativo, a perspectiva da complexidade abre novas possibilidades à compreensão do fenómeno educativo e do sujeito a educar, na medida em que abre a investigação em educação à imprevisibilidade, instabilidade e à possibilidade que caracteriza e emerge da realidade educativa nas suas múltiplas dimensões e processos, oferecendo novos conceitos e instrumentos para uma abordagem mais compreensiva do campo 
educativo. Tal como a complexidade, também a educação se centra nos mecanismos e processos da transformação complexa, na mudança e na necessidade de adaptação de fenómenos sistémicos, sujeitos à autoregulação e à emergência. A teoria da complexidade pode contribuir para que educadores e investigadores possam alcançar uma melhor compreensão do modo como se pode intervir de forma efectiva e deliberada num campo que, pela sua natureza, nem sempre responde de forma previsível, de forma a explorar novas possibilidades ou possibilidades emergentes, para além das existentes. Neste sentido, deve centrar-se nos elementos e condições dinâmicas que possibilitam a emergência de determinadas abordagens e aproximações ao objecto de estudo desenvolvendo novas possibilidades interpretativas e práticas (Vázquez, 1996).

\section{Abordagens emergentes no campo das Ciências Cognitivas e das Ciências da Educação}

Situando-se no âmbito deste quadro teórico de referência, a perspectiva que se defende inscreve-se em dois eixos de análise que se cruzam e que estruturam a perspectiva epistemológica que aqui se propõe.

Em primeiro lugar, os progressos feitos nas últimas décadas no campo das Ciências Cognitivas sobre o entendimento do funcionamento do cérebro humano. A investigação em Inteligência Artificial, em Biologia Evolutiva e em Neurociências Cognitivas gerou um novo modelo cognitivo, o qual reconhece a complexidade dos mecanismos de funcionamento e desenvolvimento cognitivo, assim como a existência de diferentes dimensões cognitivas, que têm sido tradicionalmente ignorados ou desvalorizados tanto na investigação científica como na prática educativa. Alguns destes mecanismos são as emoções e os sentimentos (Damásio, 2001) e os aspectos biológicos e sociais da cognição (Maturana, Lópes, Pérez, \& Santos, 2003; Varela, Thompson, \& Rosh, 1997). Das investigações nestes domínios, emerge a evidência da complexidade e plasticidade do cérebro e seu funcionamento, tanto em termos evolutivos relacionados com a espécie, como em termos do desenvolvimento de cada indivíduo ao longo da vida. Entende-se o cérebro como um órgão dinâmico capaz de aprender e mudar ao longo de toda a vida. Ao mesmo tempo, dados relevantes de investigações recentes mostram que a aprendizagem e os processos educativos formais podem modificar a 
organização funcional e estrutural do cérebro (Castro-Caldas, 1998), revelando a importância das experiências e oportunidades de aprendizagem para o seu desenvolvimento. Estes dados fornecidos pela investigação científica estão no centro do interesse crescente e na base de experiências recentes de colaboração entre Ciências Cognitivas e Ciências da Educação, especialmente no que diz respeito às Neurociências Cognitivas, assim como no interesse da comunidade científica na exploração das possibilidades de tal colaboração. São exemplo deste interesse iniciativas de distintas organizações como o projecto CERI da OCDE, a Harvard Graduate School of Education, o Learning Lab Denmark, entre outros, que têm desenvolvido esforços no sentido de criar pontes entre as disciplinas, promover investigação aplicada à educação e formar professores nestes domínios. Também têm surgido algumas publicações que revelam a mesma preocupação e que pretendem tornar a investigação científica disponível para o público em geral e para os educadores em particular, como é o caso das publicações da OCDE no âmbito do referido projecto (OCDE, 2002, 2007) ou de publicações de autores como Utah Frith e Susan Blackmore (2007), ou Brent Davis (2008, 2004), entre outros.

Em segundo lugar, no campo da teoria da educação destaca-se a orientação progressiva no sentido de um entendimento mais integrador do sujeito da educação (Bernal, 2002, 2005; García Carrasco, 2007) e da problemática relacionada com o desenvolvimento das suas capacidades que acentua cada vez mais a forte base biológica desta ciência, entendida como ciência da aprendizagem. Estas perspectivas têm-se preocupado em incorporar as contínuas descobertas no campo das investigações sobre o cérebro reescrevendo e (re)situando algumas das teorias do desenvolvimento e da aprendizagem que tradicionalmente estavam na sua base, introduzindo o conceito de complexidade aplicado à perspectiva sobre o funcionamento do cérebro humano, a cultura e a educação (Asensio, 1997), daí resultando enfoques inovadores que permitem um tratamento das questões transversais que atravessam a educação e que fundam a pedagogia como ciência complexa (Vázquez, 1996; Colom, 2000; Jörg et al., 2007).

No âmbito de investigação desenvolvida anteriormente, sobre modelos da cognição dominantes no domínio da intervenção cognitiva (Gonçalves, 2008), a necessidade de incluir os novos conhecimentos sobre o cérebro e 
sobre o funcionamento e desenvolvimento cognitivos tornou-se evidente, no sentido de encontrar teorias mais compreensivas, capazes de dar conta da complexidade do fenómeno da cognição e dos processos de aprendizagem que possam estar na base de propostas de intervenção mais eficazes. No nosso país, destacam-se as lacunas existentes neste domínio pela ausência de abordagens inter ou transdisciplinares sobre estas questões e da sua inclusão na agenda das prioridades ao nível da investigação em educação, assim como no âmbito da formação de professores, tal como se refere no estudo do CNE intitulado "A educação das crianças dos 0 aos 12 anos" (CNE, 2008, p. 26):

Muitas práticas pedagógicas não têm incorporado aquilo que a investigação nos diz acerca das crianças: a sua enorme capacidade para aprender, em áreas e domínios vastos (sociais, emocionais, cognitivos, linguísticos, motores, etc.); a assumpção de que a aprendizagem assenta em domínios sociais, afectivos e experienciais; a importância das interacções com famílias, entre crianças, entre educadores/professores; as janelas de oportunidade que existem para certo tipo de aprendizagens durante os primeiros anos (desenvolvimento comunicacional, competências sociais e de cidadania activa, curiosidade acerca do mundo, organização...).

Os conhecimentos já existentes sobre o cérebro humano e seu funcionamento abrem um novo universo de possibilidades no entendimento do sujeito, da cognição e da educação. A interrogação sobre a dimensão e a natureza dessas possibilidades corresponde, em larga medida, à questão expressa por Malabou (2004, p. 135): "Que novos horizontes abrem então os novos cérebros, os novos teóricos do cérebro?", principalmente no que se refere a um novo entendimento do sujeito da educação. Este sujeito - que é plástico $^{6}$ e multidimensional ${ }^{7}$ - é complexo.

Se o sujeito a educar é um sujeito bio-psico-social, as Ciências da Educação não devem ignorar nenhuma das suas dimensões. A formação do sujeito integrado (por oposição ao sujeito cindido da modernidade) não pode ser efectiva sem um claro conhecimento e investimento no desenvolvimento de todas estas dimensões. Entende-se que estes factos justificam uma abordagem como a que aqui se propõe. 


\section{A relação entre Ciências da Educação e Ciências Cognitivas}

Considera-se, então, que o surgimento de um novo campo do saber constituído pelas Ciências da Educação e as Ciências Cognitivas está relacionado com: a) a emergência de um sujeito entendido como plástico e multidimensional; b) as novas perspectivas sobre a cognição entendida como 'situada' e corporificada, em que os aspectos emocionais, sociais e biológicos representam um papel determinante; c) a necessidade de incorporar uma teoria da mudança que permita lidar com as múltiplas questões e problemas com que se confronta a prática pedagógica, que tornam necessários novos paradigmas de investigação e novas formas de colaboração que ultrapassem as barreiras entre os saberes.

Aplicando os conceitos de emergência e convergência ${ }^{8}$, utilizados por Mario Bunge (2004) para explicitar a necessidade de relação entre os distintos saberes, à realidade educativa, poder-se-á afirmar que a complexidade da realidade emergente da educação e do conhecimento do funcionamento cerebral implica a convergência de distintas disciplinas no sentido de abordar os problemas emergentes no presente. Reconhecer a natureza sistémica de muitos dos problemas educativos do nosso tempo implica defender e promover a convergência de vários saberes na tentativa de explicação e resolução desses mesmos problemas. No caso concreto da neurociência cognitiva, tomando em consideração que ela originou alguns conceitos ontológicos chave para o entendimento dos processos educativos, do sujeito da educação e mesmo das instituições educativas - como 'plasticidade neuronal', 'base neuronal dos processos mentais' ou 'arquitectura integrada em paralelo' -, pode facilmente admitir-se a necessidade da convergência entre disciplinas que se tem vindo a defender, no sentido de alcançar um melhor entendimento da realidade emergente.

Entendidas neste contexto, as Ciências da Educação devem depender de muitas outras disciplinas e interdisciplinas porque: o cérebro é plástico e é capaz de aprender ao longo de toda a vida; o sujeito que aprende é multidimensional e complexo; o sujeito tem uma vida mental e está inserido em redes sociais complexas e mutáveis; os seres humanos ocupam diferentes níveis de organização, desde físicos a sociais, e por isso não podem ser entendidos apenas num só nível; as Ciências da Educação 
necessitam de uma base científica que fundamente a investigação e suporte a actividade dos professores, as suas técnicas e estratégias de intervenção; a investigação educativa é transversal e, como tal, exige um esforço considerável, equipas de trabalho eficientes e resultados efectivos, que sejam reconhecidos socialmente e apoiados pela administração e pelos governos.

Apesar de alguns cepticismos existentes relativamente aos contributos e possibilidades que a investigação científica pode oferecer à teoria da educação (Bruer, 1995, 1997), existem algumas iniciativas que devem ser tomadas em consideração. Alguns exemplos são: o reconhecimento crescente, por parte dos investigadores da área das Ciências da Educação, da importância da investigação sobre o cérebro nas Neurociências (OCDE, 2002, 2007; Davis et al., 2008); o crescente interesse dos neurocientistas na investigação sobre os processos de aprendizagem e na aplicação das novas descobertas (Peters, 2011); o surgimento recente de iniciativas, publicações e organizações que trabalham com o objectivo de criar pontes entre as disciplinas em questão: CERI - OCDE; Cambridge University - UK (Centre for Neuroscience and Education); Bristol University (Neuro-Educational Research Network); Harvard Graduate School of Education (Mind, Brain and Education Program); Transfer Centre for Neuroscience and Learning (UIm, Alemanha), entre outros.

O reconhecimento da complexidade da realidade educativa e as profundas transformações sociais, tecnológicas e políticas verificadas nos últimos anos mostraram a insuficiência dos paradigmas existentes no campo educativo, na medida em que a sua base fisicista, ou o seu carácter linear, negligencia a complexidade inerente à realidade educativa e, por isso, não oferece uma explicação ou compreensão em profundidade dessa realidade. A dimensão dos desafios que enfrentam os sistemas educativos actuais, juntamente com os progressos alcançados por algumas ciências no que diz respeito ao funcionamento e desenvolvimento do cérebro e aos processos de aprendizagem, evidenciam a necessidade de uma estreita colaboração entre disciplinas. Essa colaboração deve estar na base de uma aproximação sistémica às questões educativas e oferecer os conceitos e ferramentas necessários para empreender as mudanças educativas pertinentes.

No documento da OCDE Understanding the brain: towards a new learning science, publicado em 2002, a questão é apresentada da seguinte 
forma: "Quanto mais aprendemos sobre o cérebro humano, especialmente no que se refere à infância (aos primeiros anos), menos confortáveis nos sentimos com o modelo tradicional de sala de aula e do currículo imposto da educação formal" (OCDE, 2002, p. 14). A complexidade emergente exige novas instituições, currículos, métodos e conhecimentos. Como refere o documento em questão, deslindar as complexidades do cérebro humano, entender a natureza da memória e da inteligência e o que acontece exactamente quando ocorre a aprendizagem são alguns dos principais objectivos que podem ser alcançados através da colaboração entre Ciências da Educação e Ciências Cognitivas. Entende-se, para além disso, que essa colaboração pode ser fundamental para refundar a prática da educação numa sólida teoria da aprendizagem: "Nomeadamente podem projectar uma nova luz sobre velhas questões sobre a aprendizagem humana e sugerir novas formas através das quais a oferta educativa e a prática de ensinar possam ajudar mais aprendizes jovens e adultos" (OCDE, 2002, p. 27). Neste sentido, tal colaboração pode também representar uma oportunidade para reorganizar as instituições educativas e repensar prioridades de investigação.

Como afirma Vázquez (2003, p. 41), "(...) a teoria da educação há-de constituir-se como uma das ciências da aprendizagem, com forte base biológica, na qual se vão incorporando as contínuas descobertas no campo das investigações sobre o cérebro humano". Esta teoria estará ligada ao surgimento de novas teorias sobre a aprendizagem humana nas suas distintas fases: infância, adolescência, maturidade, velhice, e em distintos âmbitos: matemática, literacia, entre outros (OCDE, 2002, 2007).

\subsection{Possibilidades emergentes dessa relação}

Entende-se que na base da construção da teoria da educação deve estar a complexidade da realidade educativa. Reconhecer esta complexidade e pretender intervir e reflectir sobre ela e a partir dela implica abrir novas vias para o pensamento, criar novos instrumentos para interpretar o real e integrar novas linguagens e discursos complementares, mais além de dualismos e reducionismos. Pensá-la a partir da perspectiva da possibilidade, fundada no campo da experiência vital dos sujeitos, requer, para além do mais, criar um espaço epistemológico para o qual possam convergir diferentes contributos e abordagens e a partir dos quais possam ser pensadas as dramáticas 
transformações do espaço social e cultural em que vivem e convivem os sujeitos e no qual ocorrem evoluções culturais e tecnológicas. A sua lógica, longe de ser linear (acumulativa) traduz-se num fenómeno exponencial (autoamplificador), cujo alcance e consequências não podem ser vistos a partir de perspectivas lineares ou de instituições formais mal preparadas para lidar com a incerteza e a imprevisibilidade. Dentro deste marco conceptual, entende-se, com Jörg et al. (2007), que a relação entre Ciências Cognitivas e Ciências da Educação é de natureza transdisciplinar e interdiscursiva.

Um sistema complexo pode ser definido como: composto por um grande número de componentes em interacção (agentes, processos, contextos, etc.); cuja actividade é não-linear; que é mais do que a soma das componentes individuais; e que se caracteriza pela auto-organização (Rocha, 1999). Considera-se que pensar no quadro do paradigma da complexidade implica pensar dentro de uma dinâmica não-linear e no quadro de relações, sobre estruturas e formas dos processos, e na estrutura da causalidade ao longo do tempo (epigénese). Neste sentido, trata-se de uma noção suficientemente abrangente, flexível e articuladora que gira em torno da tendência humana para pensar sob a forma da associação, preservando, e não dissecando, a integridade dos fenómenos estudados ao nível da sua emergência.

Entende-se aqui transdisciplinaridade como unificação através da fusão (Bunge, 2004), e não da redução de diferentes procedimentos, métodos e dados científicos, o que implica também novas metodologias e novas instituições de investigação - daí a dimensão prática da perspectiva em causa. Este enfoque funda-se em teorias da complexidade, utiliza instrumentos de pensamento transdisciplinares e incorpora uma teoria da mudança, para entender a evolução dos sistemas complexos. Por interdiscursividade entende-se a necessidade de conjugar diferentes discursos para um melhor entendimento das possibilidades humanas.

Através desta perspectiva torna-se possível pensar a realidade da aprendizagem e da educação em termos de espaços de possibilidade, na linha do definido por García Carrasco (2003), inspirado em Vigotsky, como zona de construção do sujeito, e de potencialidades de aprendizagem e desenvolvimento para os aprendizes: "Os complexos e tortuosos caminhos da aprendizagem podem ser retratados como trajectórias temporais de 
aprendizagem nesses espaços transitórios multidimensionais" (Jörg et al., 2007). Trata-se, em suma, de reinventar e transformar a descrição e explicação do fenómeno da aprendizagem numa nova e mais rica realidade. Neste sentido, o que se pretende é fundar uma ciência da aprendizagem mais compreensiva, baseada num novo léxico da complexidade e que inclua conceitos transversais como interactividade, conectividade, generatividade, complexidade, entre outros.

Existem, neste campo, alguns riscos e obstáculos a ter em conta na construção de tal teoria. Alguns dos mais relevantes estão relacionados quer com as condições de emergência da reflexão e da prática da transversalidade no campo concreto da relação entre Ciências da Educação e Ciências Cognitivas, quer com a forma como os diferentes discursos e práticas ou modelos de investigação podem ser conjugados.

Como referido noutro lugar (Gonçalves, 2009), no quadro dessas dificuldades ou obstáculos, a possibilidade de uma abordagem transdisciplinar dos fenómenos educativos depende da capacidade de: integrar o novo léxico da complexidade e criar um léxico comum, definindo e integrando conceitos das diferentes ciências; estabelecer novas prioridades e instituições de investigação; abrir o debate a outras disciplinas, distinguir o que está bem estabelecido, o que é provável e o que constitui um mito sobre o funcionamento do cérebro e os processos de aprendizagem; promover a aplicação prática do conhecimento neurocientífico; promover a investigação baseada nos problemas originários da prática educativa e da pedagogia; estabelecer uma metodologia comum.

Dificuldades de outra natureza estão relacionadas com a defesa da criação de uma nova ciência da aprendizagem, a qual se considera que é susceptível de levantar algumas questões que é necessário ter em conta. Tanto os documentos da $\operatorname{OCDE}(2002,2007)$ que têm vindo a ser referidos, como as perspectivas de Jörg et al. (2007), relacionam a abordagem transdisciplinar da educação com o surgimento de uma nova ciência da aprendizagem, mais compreensiva e integradora dos diferentes contributos disciplinares com ela relacionados. No entanto, nem sempre a forma como é abordada e apresentada essa nova ciência corresponde a uma abordagem complexa e sistémica dos fenómenos em causa. Neste sentido, será necessário chamar a atenção para o perigo de alguma precipitação neste campo e para os riscos de simplificações redutoras. 
Partindo da consciência da complexidade da realidade educativa e da natureza sistémica do funcionamento cerebral e mental dos indivíduos, considera-se que o termo 'transdisciplinaridade', como contraponto do termo mais comum 'interdisciplinaridade', representa mais uma refundação ou renovação da teoria da educação como teoria da complexidade. Como tal, deve ser referida a uma atitude de investigação entendida como abertura do campo de investigação educativa a diferentes discursos que permitam e fundamentem cientificamente uma visão mais integradora do sujeito da educação e que vão progressiva, mas cautelosamente, contribuindo para pensar os aspectos e dimensões em que essa colaboração se pode tornar efectiva e frutífera.

A transdisciplinaridade é, neste sentido, entendida como forma de investigação, que confronta e faz convergir num mesmo projecto diferentes perspectivas e preocupações disciplinares em função da resposta a questões que são comuns. Partindo desta perspectiva prática, pode falar-se de uma nova ciência da aprendizagem que evite alguns riscos de redução, presentes em termos ou conceitos como 'brain-based-learning' ou 'brain-basededucation'. Estes termos podem ser entendidos como mera programação neuronal ou cognitiva e simplificados e instrumentalizados - o que parece estar já a acontecer em alguns casos. Uma perspectiva transdisciplinar da educação, entendida desta forma, possibilitará e fomentará o surgimento de estudos transdisciplinares e o seu desenvolvimento e multiplicação no futuro.

\section{Conclusões}

Um modelo baseado no paradigma da complexidade e no modelo do funcionamento cerebral, como órgão por excelência da complexidade, recusa necessariamente perspectivas redutoras sobre a relação entre as ciências e entende a relação das neurociências com a educação como uma relação bidireccional, que oferece bases para uma sólida teoria da aprendizagem, organizado em torno de questões fundamentais e orientado pela e para a prática educativa.

Esta viragem epistemológica representaria a superação de algumas dicotomias que têm condicionado a reflexão sobre a educação: a dicotomia teoria/prática, vistas como duas noções antagónicas, e a dicotomia 
natureza/cultura, normalmente tematizadas como genética versus formação do comportamento através das influências do meio. A superação da primeira torna-se, não só possível, como necessária, na medida em que se entendem como duas formas de prática que integram, ambas, actividade prática e actividade de reflexão (García Carrasco, 2003). A segunda é superada pelo novo entendimento do sujeito enquanto unidade psicossomática proporcionado pela decifração do genoma humano (com todas as suas limitações) e pelo progresso das neurociências.

A complexidade sistémica instaura uma série de níveis de análise necessários para a compreensão do ser humano enquanto sujeito individual, social, comunicacional e como multidimensional, tanto ao nível biopsicológico, como cultural e social, em que é necessário ter em conta as relações entre os diferentes elementos e níveis do sistema. Ao mesmo tempo, o sujeito como sujeito da plasticidade, que se constrói com e através da sua história, em contextos culturais e sociais concretos e segundo uma temporalidade própria, implica uma teoria da mudança que seja compreensiva relativamente aos processos de construção do sujeito em todas as suas dimensões, e de acordo com as variáveis espaço e tempo. A compreensão da trama em que se tece a construção do sujeito necessita ser entendida, por um lado, nas práticas de uma comunidade e, por outro, através de comunidades de prática investigadora, ou comunidades de investigação. Entende-se que a forma como se desenvolverem os trabalhos e como evoluir este tipo de organizações será decisiva para a investigação transdisciplinar e definirá o futuro da investigação aplicada no campo das Ciências Cognitivas e das Ciências da Educação. Permitirá também pensar e fomentar novas formas de organização e de colaboração.

Promover o desenvolvimento de iniciativas que permitam fomentar o trabalho colaborativo e criar pontes entre as disciplinas, que funcionem como mecanismos que permitam avançar no estudo da mente e do cérebro e também da educação, apresenta-se como uma necessidade. A colaboração poderá ser benéfica tanto para educadores como para neurocientistas, na medida em que podem explorar novas perspectivas e alargar o horizonte a partir do qual podem ser colocadas e respondidas questões importantes sobre a forma como aprendem os seres humanos. Será importante discutir-se os mecanismos necessários para promover essa colaboração e definir os 
âmbitos em que pode vir a ser aplicada proximamente. Algumas áreas, como a formação de professores (Ansari, Coch, \& Smedt, 2011), de investigadores (OCDE, 2007) e o estabelecimento de novas iniciativas de colaboração, devem ser prioritárias (Gonçalves, 2009). A conjugação de metodologias diversas e de múltiplos níveis de análise em diferentes contextos deve ser integrada por numa abordagem compreensiva e transdisciplinar dos fenómenos em estudo. A discussão sobre as distintas perspectivas metodológicas e conceptuais implicadas nessa colaboração poderá constituir um importante instrumento para refinar e redefinir o papel e natureza das ciências no futuro e determinar a necessidade de uma nova ciência que conjugue as diversas dimensões e perspectivas.

Recentemente, o desenvolvimento e consolidação de um campo interdisciplinar designado como "Educational Neuroscience" tem sido reconhecido progressivamente. Segundo Patten e Campbell (2011), este campo envolve sínteses de teorias, métodos e técnicas das neurociências, enquanto aplicadas e enquadradas pela investigação e prática educativas, que inspira e é inspirado por questões e problemas emanados do contexto educativo. As investigações desenvolvidas nesta área têm tido aplicações em âmbitos educativos tão diversos como o ensino da matemática, a literacia, a formação de professores, o design de ambientes de aprendizagem, entre outros. Existe uma grande diversidade de investigações e perspectivas neste domínio, assim como de iniciativas desenvolvidas. No entanto, o objectivo último deste campo de investigação é o de contribuir para melhorar o ensino e a aprendizagem. Formar uma nova geração de profissionais e investigadores em educação e reconciliar as novas descobertas em neurociências com as teorias estabelecidas, seja através da validação de práticas pedagógicas vigentes ou da promoção de novas práticas, constitui um desafio que deve conjugar esforços de todas as áreas científicas envolvidas. Ajudar os professores a tornarem-se conhecedores das questões e conhecimentos das neurociências e suas implicações nas suas práticas profissionais, assim como contribuir para que os neurocientistas se familiarizem com as questões e problemas relacionados com a prática de sala de aula, são das tarefas mais importantes a desenvolver no futuro.

A Biologia Evolutiva (Lewontin, 1998) e do Conhecimento (Capra, 1998) podem também representar um contributo importante para repensar as 
questões evolutivas relacionadas com a cognição e a aprendizagem, ultrapassando a ideia tradicional de um desenvolvimento 'natural', linear e previsível, cuja influência continua a fazer-se sentir em educação. Estes domínios do saber configuram abordagens sistémicas e complexas do desenvolvimento fundadas numa perspectiva da evolução dos sistemas vivos como sistemas complexos, isto é, auto-organizados, emergentes, abertos em suma, plásticos.

\section{Notas}

1 Designa-se por cognitivismo clásico a aproximação ao estudo da mente que tem como base o modelo simbólico assente na teoria do processamento da informação. Este modelo entende a mente como um programa de computador que manipula símbolos. O computador aparece neste modelo como metáfora explicativa da mente humana (Crespo, 2002).

2 O conceito de paradigma é aqui utilizado no sentido de um programa de investigação, que parte de uma determinada visão de mundo ou ponto de vista, para criar um núcleo de inteligibilidade e fornecer instrumentos a partir dos quais analisar os múltiplos fenómenos ou dimensões do real. As mudanças de paradigma ocorrem normalmente quando a atenção se foca em fenómenos que não podem ser explicados de forma satisfatória pelas teorias dominantes. Acontece quando alguns fenómenos emergentes captam o interesse e assumem progressiva importância no seio de uma comunidade científica e as alternativas propostas demonstram um valor acrescido na medida em que oferecem uma melhor explicação do que o modelo teórico dominante (Camras \& Witherington, 2005). Entende-se paradigma, no sentido que Ihe atribui Morin (1992), como um conjunto de relações fundamentais de associação e/ou oposição dentro de um número restrito de noções mestras - noções estas que comandam ou controlam todos os pensamentos, discursos e teorias.

3 A teoria dos sistemas dinâmicos é originária dos estudos biológicos sobre os organismos vivos e sua evolução, da teoria do caos e de uma perspectiva nãolinear do desenvolvimento que estuda os processos de mudança (e não de crescimento) a partir de uma perspectiva probabilística, tendo em conta que o indivíduo está imerso em múltiplos contextos - sociais, ecológicos, culturais, biológicos - em permanente mudança, dentro dos quais a estabilidade, e não a mudança, é a excepção à norma. A perspectiva sistémica é entendida como uma metateoria (Gutiérrez, 2005) ou um conceito-ponte (Bunge, 2004), na medida em que é susceptível de ser aplicada ao estudo de diversos fenómenos (biológicos, psicológicos, sociais) e deve servir de referência à reflexão no âmbito da complexidade. O pensamento sistémico funciona em termos de conexões, relações e contexto. A abordagem sistémica ao estudo da cognição assenta na convicção de que o funcionamento cognitivo é próprio de um sistema altamente organizado, embora não fechado, cujos componentes interagem uns com os outros de forma 
complexa, afectando cada um a aplicação e desenvolvimento dos outros, pelo que se torna impossível abordar qualquer um deles em profundidade sem fazer referência aos restantes processos (Gutiérrez, 2005). Entende-se ainda que esse sistema funciona em diferentes níveis interligados entre si: físico, cognitivo, emocional, social.

4 Transdisciplinaridade: termo que serve para designar uma investigação que se caracteriza pela unificação de diferentes linhas de investigação e procura estudar um fenómeno nas suas diferentes dimensões e níveis de emergência, preservando a sua integridade. A transdisciplinaridade complementa tanto a pluridisciplinaridade (o estudo de uma determinada disciplina através da utilização da visão de outras disciplinas) como a interdisciplinaridade (a transferência de métodos adoptados por uma determinada disciplina para outra), no sentido de apreender os diferentes níveis de uma realidade multidimensional de uma forma sistémica e complexa (Nicolescu, 1996; OCDE, 2007).

5 Interdiscursividade: a conjugação de diferentes discursos para uma melhor compreensão das possibilidades humanas. Implica o reconhecimento da incapacidade de um discurso isolado poder dar conta de fenómenos emergentes (Jörg, Davis e Nickmans, 2007; Davis, 2008).

6 Em termos neurocientíficos, a plasticidade neuronal é um termo complexo que envolve distintos factores, níveis e condicionantes, cujos traços ou implicações principais se considera que poderiam ser organizados da seguinte forma: a) 0 cérebro tem a capacidade de mudar, adaptar-se e, logo, de aprender ao longo de toda a vida. A plasticidade subjaz à aprendizagem; b) $\mathrm{O}$ cérebro muda como resposta à estimulação ambiental, o que quer dizer que não está completamente determinado à partida. Essas mudanças são determinadas pela interacção de factores genéticos e epigenéticos; c) A plasticidade implica periodicidade, na medida em que existem períodos sensíveis ou 'janelas se oportunidade' que favorecem ou dificultam determinadas mudanças; d) A plasticidade está sujeita a limites relacionados com o funcionamento e organização cerebral (neurogénese e apoptose); e) O funcionamento do cérebro é plástico e a sua organização é integrada; f) $O$ uso que se faz do cérebro é determinante para o seu desenvolvimento, para a sua 'construção'. Entendida como um conceito complexo, a plasticidade tem implicações que transcendem os limites da ciência e colocam questões educativas, sociais ou mesmo políticas.

7 A ideia de multidimensionalidade surge aqui ligada à ideia de complexidade. Neste sentido, entende-se por sujeito multidimensional um sujeito bio-psico-social, que é uma construção bio-antropológica, cuja identidade não é monolítica, uma vez que se constrói entre distintas e diferentes dimensões interligadas entre si, e à qual subjaz a unidade de uma consciência. Como afirma Edgar Morin (1999: 13), "Las unidades complejas, como el ser humano o la sociedad, son multidimensionales; el ser humano es a la vez biológico, psíquico, social, afectivo, racional", e ser complexo implica precisamente a unidade dessas várias dimensões: "Complexus significa lo que está tejido junto; en efecto, hay complejidad cuando son inseparables los elementos diferentes que constituyen un todo (como el económico, el político, el sociológico, el afectivo, el mitológico) y que existe un tejido interdependiente, interactivo e inter-retroactivo entre el objeto de conocimiento y su contexto, las partes y el todo, el todo y las partes, las partes entre ellas. Por esto, la complejidad es la unión entre la unidad y la multiplicidad" (Morin, 1999, p. 14). 
8 Emergência é, segundo Mário Bunge (2004), uma categoria ontológica que alude à origem de novidades e implica divisão, divergência; está relacionada com a aparição de uma novidade qualitativa e relacionada com termos como "sistema", "auto-organização", "caos", "fractal", "complexidade", "módulo", "consciência". Convergência é uma categoria epistemológica que implica a fusão entre linhas de investigação inicialmente separadas, implica transdisciplinaridade, "unificação", "fusão" ou "integração". Segundo o autor, as condições formais necessárias para a emergência de uma transdisciplina acontecem quando se demonstra o valor heurístico de fundir enfoques de investigação inicialmente independentes. As duas categorias não são, por isso, mutuamente excludentes, na medida em que a compreensão da emergência (novidade) exige frequentemente a convergência de duas ou mais linhas de investigação.

\section{Referências}

Ansari, D., Coch, D., \& Smedt, B. (2011). Connecting education and cognitive neuroscience: Where will the journey take us? Educational Philosophy and Theory, 43(1), 37-42.

Asensio, J. M. (1997). Biología y educación: El ser educable. Barcelona: Ariel.

Bernal, A. (2002). Reconceptualización de la idea de sujeto y educación. In A. Bernal (Coord.), Persona, curriculum y postmodernidad (pp. 25-62). Barcelona: PPU.

Bernal, A. (2005). Reconceptualización de la identidad personal y educación para la autodeterminación posible. Teoría de la Educación, 17, 97-128.

Bruer, J. T. (1995). Escuelas para pensar: Una ciencia del aprendizaje en el aula. Barcelona: Ediciones Paidós.

Bruer, J. T. (1997). Education and the brain: A bridge too far. Educational Researcher, 26(8), 4-16.

Bunge, M. (2004). Emergencia y convergencia: Novedad cualitativa y unidad del conocimiento. Barcelona: Gedisa.

Camras, L. A., \& Witherington, D. C. (2005). Dynamical systems approaches to emotional development. Developmental Review, 25, 328-350.

Capra, F. (1998). La trama de la vida. Barcelona: Anagrama.

Castro-Caldas, A. (1998). The illiterate brain. Learning to read and write during childhood influences the functional organization of the brain. Brain, 121, 10531063.

Colom, A. J. (2000). Desarrollo sostenible y educación para el desarrollo. Barcelona: Octaedro.

Conselho Nacional de Educação (CNE) (2008). Relatório do estudo "A educação das crianças dos 0 aos 12 anos". Lisboa: Conselho Nacional de Educação.

Crespo, A. (2002). Cognición humana. Mente, ordenadores y neuronas. Madrid: Ediciones Centro de Estudios Ramón Areces. 
Cruz, V., \& Fonseca, V. (2002). Educação cognitiva e aprendizagem. Porto: Porto Editora.

Damásio, A. (2001). O sentimento de si: O corpo, a emoção e a neurobiologia da consciência. Mem Martins: Publicações Europa-América.

Davis, B. (2004). Inventions of teaching. A genealogy. New York/London: Routledge.

Davis, B. (2008). Complexity and education: Vital simultaneities. Educational Philosophy and Theory, 40(1), 46-61.

Davis, B., Sumara, D., \& Luce-Kapler, R. (2008). Engaging minds. Changing teaching in complex times. New York/London: Routledge.

Dortier, J. F. (Coord.). (2003). Le cerveau et la pensée. Auxerre: Éditions Sciences Humaines.

Dumont, H., Istance, D., \& Benavides, F. (Ed.). (2010). The nature of learning. Using research to inspire practice. Paris: OCDE.

Flores, M. A., \& Day, C. (2006). Contexts which shape and reshape new teachers' identities: A multi-perspective study. Teaching and teacher education, 22, 219232.

Frith, U., \& Blakemore, S. (2007). Como aprende el cerebro. Las claves para la educación. Barcelona: Ariel.

García Carrasco, J. (2003). Evolución del contexto social y cambios en el pensamiento sobre la educación. Educación por la influencia o educación desde la experiencia. In Teoria de la educación ayer y hoy (pp. 70-114). Murcia: Seminario Interuniversitario de Teoría de la Educación.

García Carrasco, J. (2007). Leer en la cara y en el mundo. Barcelona: Herder Editorial.

Gazzaniga, M. S. (2004). The cognitive neurosciences. Cambridge: MIT Press.

Gonçalves, T. (2008). Educação e cognição. Um modelo de análise de programas de desenvolvimento cognitivo. Tese de doutoramento apresentada à Universidade de Sevilha [documento policopiado].

Gonçalves, T. (2009). Complex systems and plastic brains. A trans-disciplinary approach to education and the cognitive sciences. In M. Karanika-Murray \& R. Wiesemes (2009), Exploring avenues to interdisciplinary research: From cross- to multi- to interdisciplinarity (pp. 9-24). Nottingham: Nottingham University Press.

Gutiérrez, F. (2005). Teorías del desarrollo cognitivo. Madrid: McGraw-Hill.

Jörg, T., Davis, B., \& Nikmans, G. (2007). Towards a new, complexity science of learning and education. Educational Research Review, 2(2), 145-156.

Lewontin, R. (1998). A tripla hélice. Gene, organism e ambiente. Lisboa: Edições 70.

Malabou, C. (2004). Que faire de notre cerveau? Paris: Bayard.

Maturana, H., López, M., Pérez, A., \& Santos, M. (2003). Conversando con Maturana de educación. Málaga: Ediciones Aljibe.

Morin, E. (1992). From the concept of system to the paradigm of complexity. Journal of Social and Evolutionary Systems, 14(4), 371-385.

Morin, E. (1999). Los siete saberes necesarios a la educación del futuro. Paris: UNESCO. 
Nicolescu, B. (1996). La transdisciplinarité - Manifeste. Mónaco: Editions du Rocher. OCDE (2002). Understanding the brain. Towards a new learning science. Paris: OCDE. OCDE (2007). Understanding the brain. The birth of a learning science. Paris: OCDE.

Osberg, D., Biesta, G., \& Cilliers, P. (2008). From representation to emergence: Complexity's challenge to the epistemology of schooling. Educational Philosophy and Theory, 40(1), 213-227.

Patten, K., \& Campbell, S. (2011). Introduction: Educational Neuroscience. Educational Philosophy and Theory, 43(1), 1-6.

Peters, M. A. (Ed.). (2011). Educational Philosophy and Theory, 43(1), 1-107.

Rocha, L. M. (1999). Complex systems modeling: Using metaphors from nature in simulation and scientific models. Disponível em http://informatics.indiana.edu/rocha/complex/csm.html (acesso em Maio de 2010).

Roldão, M. C. (2005). Saber educativo e culturas profissionais: Contributos para uma construção-desconstrução epistemológica. In Actas do VIII Congresso da SPCE, "Cenários de educação/formação: Novos espaços, culturas e saberes". CD- ROM.

Roldão, M. C. (2007). Função docente: Natureza e construção do conhecimento profissional. Revista Brasileira de Educação, 34, 94-103.

Varela, F. J., Thompson, E., \& Rosch, E. (1997). De cuerpo presente. Barcelona: Gedisa.

Vázquez, G. (1996). La pedagogía como ciencia de la complejidad. Medellin: Universidad Pontifícia Bolivariana.

Vázquez, G. (2003). Aprender y pensar. Visión in(re)trospectiva del componente tecnológico del SITE. In Teoría de la educación ayer y hoy (pp. 20-68). Murcia: Seminario Interuniversitario de Teoría de la Educación. 
EDUCATIONAL SCIENCES AND COGNITIVE SCIENCES. A TRANS-DISCIPLINARY APPROACH

\section{Abstract}

The development of scientific research in the field of Cognitive Sciences contributed to a newer and more complex perspective about the human subject, learning and cognition. The nature of the educational phenomenon and its fast transformations point out for the need to incorporate a theory of change allowing to deal with the multiple questions and problems in the field of education. Considering complexity theory as an emergent paradigm in the field of Natural Sciences as well as in Social Sciences, some arguments for the cooperation between Educational Sciences and Cognitive Sciences will be presented, clarifying its need and implications, as well as the possibilities that it will represent to educational research and action.

Keywords

Education; Cognition; Complexity; Transdisciplinarity

\section{CIENCIAS DE LA EDUCACIÓN Y CIENCIAS COGNITIVAS. UN ENFOQUE TRANSDISCIPLINARIO}

\section{Resumen}

El desarrollo de la investigación científica en el ámbito de las Ciencias Cognitivas ha contribuido para una nueva y más compleja perspectiva sobre el sujeto de la educación, la cognición y el aprendizaje. La naturaleza del fenómeno educativo y las rápidas transformaciones por las que ha pasado recientemente remeten para la necesidad de incorporar una teoría del cambio que permita afrontar las múltiples cuestiones y problemas con los que se depara la reflexión en educación y la práctica educativa. Estos aspectos tornan necesarios nuevos paradigmas de investigación y nuevos modos de cooperación que ultrapasen las barreras entre los saberes. Considerando la 
teoría de la complejidad como paradigma emergente en el ámbito de las Ciencias Naturales y las Ciencias Sociales y Humanas, se presentan algunos argumentos en defensa de la cooperación entre Ciencias de la Educación y Ciencias Cognitivas, clarificando su necesidad y implicaciones, bien como las posibilidades que representa para la investigación y la acción educativas.

Palabras-clave

Educación; Cognición; Complejidad; Transdisciplinaridad

Recebido em Fevereiro/2010 Aceite para publicação em Janeiro/2012 